\title{
Intranets: A New Dimension for Library Services
}

\author{
Bhojaraju G
}

\begin{abstract}
This article is based on the some practical experiences of Indian Rubber Manufacturing Research Association (IRMRA). It gives brief introduction about the intranet, controversies of an intranet, and its library applications. It also gives information about accessing management information, improving internal communications, e-mail, intra mail, collaborative working, communities of interests, discussion groups, electronic forms, internal newsletters, search facilities, training materials, and library applications/access. The use of intranet in providing library services like SDI, CAS and other information management is explained with a schematic diagram.
\end{abstract}

\section{INTRODUCTION}

Intranets are widely considered to be simply an internal version of the internet, a sort of miniature version of the web running on a company or institutional network. The appeal of intranets is that they provide a single central information store and encourage communication flows throughout the organisations. Information can be easily be shared and disseminated to large groups of people dispersed across different sites, even across national borders. There is potential to link every desktop in the organisation irrespective of which computing systems are in use. A vast range of internal company information can be held on the intranet. At a basic level this could include internal telephone lists, procedures, manuals, organisational charts, personnel documentation, and the type of information, which is almost impossible to keep up-to-date using hardcopy formats. More advanced information handling scenarios are also feasible.

Internet is a communication network which links all the small computer networks worldwide as one whole. Intranet is based upon internet technology, in particular world wide web (WWW), to build information systems within an organisation or enterprise to accomplish standardization and automation. Fundamentally, it means network-computing environments, which let the users share the information through the internet and web browsers. Ultimately, it allows a certain organisation to build a groupware within web environment at low cost on top of existing network infrastructure. By doing this, closed organisation network would be interconnected with existing worldwide internet, which results in diverse information that strengthens competitive advantages of the organisation. Basically, it runs on top of TCP/IP and HTTP and filters out any illegal access through firewall.

In libraries, intranet can facilitate so many services. It helps in dissemination of stored information as well as allows to access to remote information. Through intranet, libraries can disseminate required information to its users in less time, with high effectiveness. Information professionals are becoming 
webmaster and intranet coordinators, combining technical expertise with information management ability. With increasing emphasis on resource sharing not only between libraries but between different branches and departments of a library, the intranet offers the potential to be a very important tool in libraries' effort to make the most efficient use of their resources.

In Steve Gilliland's words, "The intranet is a very powerful paradigm for sharing information across the company. It works on server centric computing. There's a lower cost of application development, a lower cost of software upgrades, and it gives you ubiquitous access to information and applications... There's a browser deployed on every desktop... it accesses everything and there's no need for training."

\section{CONTROVERSIES OF AN INTRANET}

The most salient controversy is security. Security is an issue for the intranet and the internet. Security issues have to be addressed fully. Not all information can be put online; payroll, personal employee information, engineering prototypes, company secrets, and so forth. However, in order for the intranet to be fully functional, this information needs to available online. Information like this needs to be secured behind fire bridges (security that allows access only by authorised members).

The web does not handle login, security, access control, authorization, or replication very well yet. So, setting-up fire bridges like this is challenging. Issues of directory and security interoperability are significant hurdles as well. With the introduction of directory servers and new internet protocols for dealing with directories and operating system differences, this issue will be resolved. However, the maturation of the technology must take place to handle these hurdles.

\section{INTRANETS FOR LIBRARY SERVICES}

The role of information officer or librarian in the facilitation of intranet and internet solutions is a key one. This role brings the librarian into a regular contact and working relationships with the system staff and information suppliers. The librarian often remains the best judge of an information source and knows the information needs of the organisation within which he works. Thus, the librarian can provide an important liaison in order to use the intranet as a platform to disseminate the library's own materials and newsletters or as a source to bring external information into the organisation.

The intranet is perhaps our best opportunity to talk to the rest of our organisation, and provide the information centre with a valuable and effective tool for dissemination of information.

A vast range of internal company information can be held on the intranet. At the basic level this could include internal telephone directory, procedures, manuals, organisational charts, other personnel documentation, and the type of information which is almost impossible to keep up-to-date using hard-copy formats. More advanced information handling scenarios are also feasible.

As the librarian moves into a more proactive role, he offers a highly effective way of pushing information at the user. Information professionals are becoming webmaster and intranet co-ordinators, combining technical expertise with information management ability. For information services staff the intranet offers a wonderful range of technologies on which they can develop their skills as information managers.

The intranet is another step in the direction of becoming proactive as opposed to custodians of dusty old cuttings and the low status which accompanied such a role. It is not insignificant that the intranet development has been accomplished by redefining of the roles of staff and attempts to change the image of information service provider away from the passive librarian towards the dynamic research specialist.

As use of internet and intranet grows, so too will the number and kinds of application 
software. Presently, most intranet web sites are used for very basic information sharing, such as the posting of phone directories, job listings or company documents. However, more sophisticated software allow for greater linkage between databases, remote sites and the transfer of video and audio material, holding of virtual conferences around the world, all of which may be applicable to libraries.

\subsection{Library Applications}

The primary benefit of an intranet in a library is the facilitation of information sharing. Various kinds of documents can be converted into HTML and made web-accessible. Policies, procedures, forms, annual reports, manuals, schedules, calendar of events, programmes, and anything on paper can be transferred onto an intranet. The intranet will then serve as a quick and reliable source for organisational information. In fact, anything of interest or use of the staff, either professionally or personally, may be put onto it. In this way librarians and other employees, and for some information, the public can be more aware of what is going on at the library.

An intranet can also provide access to online databases such as those of DIALOG. Here, the ability of an intranet to restrict access is important. Special interfaces will be needed for Online Public Access Catalogues (OPACs) and Z39.50 gateways to access other databases. The intranet is certainly a step towards a 'virtual library'. In this context Cary Grrifiths says-"Think of an intranet as an organisation's virtual library".

\subsection{Specialised Library Services}

There are number of obvious advantages for an information and library service which has access to the parent organisation's intranet. It can publicize its services to its user community through a variety of pages, some of them generally static (opening hours, details of key staff, etc.) and some of them updated more frequently (new additions or journal alert listing service). Figure 1 shows services given by IRMRA library over internet.
An intranet can assist the information professional to develop document delivery services. Documents can be delivered to remote printers alongside users' workstations.

- Network fax and e-mail offers the possibility for externally sourced copies to be delivered to the network

- SDI services can provide abstract and article summaries by e-mail, or order bulletins to be printed at the users' terminal

- Intranet allows direct posting of the results of research on electronic databases

- The library can give access to its catalogues (OPAC) or other bibliographic database will be connected directly to the network

- Library and information services are often responsible for arranging the supply of external news feeds and other information resources on intranets.

\subsubsection{Accessing Management Information}

The library typically provides access to a large number of resources. These resources include books, journals, CD-ROM databases, online services and even the library OPAC. Usage information is an example of management information. Other examples of management information are reports and reviews written by members of staff. Management information is something that can be relatively easily delivered to librarian's desktops using off the shelf products and/or a few inhouse developed programs.

For example, one can provide access to a number of CD-ROMs over the campus network. In order to use one of these CD-ROMs, users must supply a user name and password which is validated against the users' data on the computing services central computing resources.

A webserver can be loaded on to the library OPAC machine in order to prototype the webOPAC. 


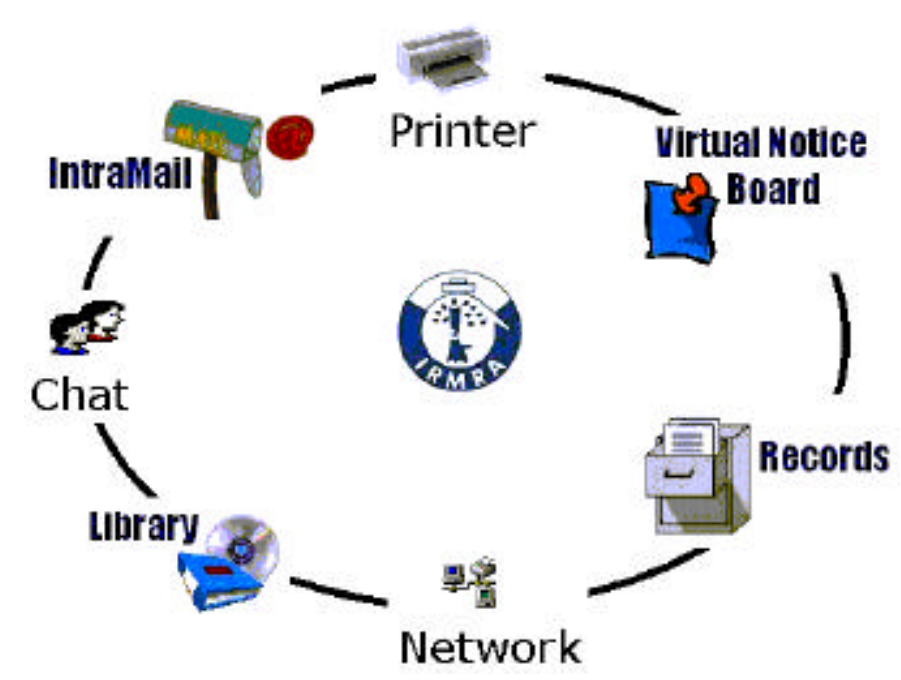

Figure 1. Library services over intranet at IRMRA

\subsubsection{Improving Internal Communication}

Along with providing a means of allowing access to existing data sources for management purposes, the intranet is also supposed to help foster group working.

\section{(a) E-Mail}

In most academic institutions, at least e-mail has already become a replacement or alternative to internal memoranda. The ability to easily set up and maintain internet mailing lists can be applied in the intranet context to support group communication. Mailing list can be archived and the archive made available from the intranet's web server to allow a group memory to be developed. Mailing list archives are also more accurate than traditional meeting minutes and means that groups no longer need to dedicate someone to make notes of their decisions.

\section{(b) IntraMail}

IntraMail (Intra E-mail) is a facility that can be used to great advantage by the college/organisation faculty and other office staff. Any member can send email to any other member of the organisation. This will reduce unnecessary paper work and improve efficiency. IntraMail complements the current intercom facility by enabling the members to communicate with each other within the organisation. It also stores messages in the mailbox. This feature will help to correspond, transfer documents and informative articles to everyone.

\subsubsection{Collaborative Working}

The ability to share documents and manage the processes of authorship through document workflow is one of the most valuable uses of an intranet in any organisation. Intranets also allow collaborative online working, for example using shared whiteboards, video conferencing, and management round tables based on discussion group technology.

\subsubsection{Communities of Interests}

Groups with a common interest can be brought together. The libraries within an organisation can provide a single virtual presence even if they are widely spread across a number of campuses or offices around the country. Forms and e-mails allow users to route enquiries to the most appropriate expert section.

\subsubsection{Discussion Groups}

These are frequently found on intranet. They offer a kind of collaborative work area. Ideas can be exchanged on a variety of topics. Discussion groups are often managed using a list server, operated using the same software that runs many internet mailing lists. A number of freely available software packages are widely used, notably Majordomo (for unix servers), Listserv and Listproc. 


\subsubsection{Electronic Forms}

Forms can be used to query databases or to provide an interface to search engines. Applications familiar form the internet, such as newsgroups, can be provided for the internal community using the intranet.

\subsubsection{Internal Newsletters}

Internal publications are often hosted on the intranet. With the digital cameras, rapid coverage of events is possible.

\subsubsection{Search Facilities}

Search engines and other software familiar from the web can be used on the internal networks and locate the various versions of a given document. There is considerable potential for the library and information service to be involved in the developing the use of the engines, as well as supporting the management of the versions, ensuring that the most accurate, authoritative and recent version of a document is retrieved when search terms are entered.

\subsubsection{Training Material}

Training material in the form of multimedia can be delivered by content often carried by intranets. A number of companies produce training videos in electronic format as CD-ROMs or DVDs. Materials produced specifically for computer-based training can often be networked. If the training material refer to publications available in the library collection, there is an opportunity for the library to provide links to the catalogue or to other information about the publications cited.

\subsubsection{Library Applications/Access}

The intranet for the library services can be used in the following ways also:

- Books for Approval: The librarian can forward list of books for approval directly to the Head of Department through e-mail, who can approve or reject them without visiting the library.

- Recommendations to the Library: The staff members can directly make requests to the librarian for purchase of particular titles via the intranet. The same can be intimated to the respective staff when the requested title has been acquired by the library.

- Department-Wise New Books Display: A list of new arrivals in the library can be displayed department-wise to keep the faculty members up to date.

- Overdue Notices: Librarian can send reminders to the defaulting members by sending intranet e-mail reminders.

- Renewal of Issued Books: Faculty members can get their books renewed without having any visit to the library.

- Electronic SDI: By maintaining 'User Profile' and 'Document Profile', one can provide E-SDI service to its users. The list of latest addition to the library, journal articles of users' interest can be scanned and sent as an attachment. Also articles of interest downloaded from the world wide web can also be saved and later on sent to the user or pasted on the bulletin board of the library page, thus saving the time required to surf the net.

- Electronic Bulletin Board:List of latest additions of books, journal issue, notices, forthcoming seminars, examination schedules, membership renewals, etc., can be given. News headlines from daily newspapers downloaded from the internet can also be posted.

- Downloads: The library can provide the Articles-Alert service to its users by downloading e-journals freely available on internet and can be uploaded on to the intranet. As the journals may be current and also difficult to subscribe or sometimes not available as hardcopy. This enables to provide an excellent current awareness service (CAS).

\section{CONCLUSION}

The most impressive contribution an intranet will make to any organisation is in its communication, coordination and collaboration benefits. The intranet has certainly revolutionised the information management processes in the libraries. Intranet, which has distinct advantage over LAN, has helped in faster data collection and dissemination. It is indeed a challenge to the 
library professionals not only to accept and assimilate this new technology, but also to spice it up with the classical knowledge to further improve the entire information management processes in the libraries. Lest we ourselves risk being obsolete. An intranet is a learning organisation, capable of integrating people, processes, procedures and principles to form an intellectually creative culture dedicated to implementing total organisational effectiveness.

Intranets are relatively cheap and easy to develop because they use the existing technology of the internet. The value of an intranet is that it can integrate in one central source, and is a combination of internal, external, formal and informal information. This is an important consideration in the corporate sector where informal information sources are stated so highly. Positive communication flows are encouraged and interaction between colleagues and project teams is seen as a valuable way of creating a shared knowledge base for the organisation. There is a suggestion however that because intranets are productive layer in knowledge economy engineered organisations' they are less likely to be really successful in strictly hierarchical organisational structures.

For information services staff, the intranet offers a wonderful range of technologies on which they can develop their skills as information managers. Information professionals are becoming webmaster and intranet coordinators, combining technical expertise with information management ability. As the librarian emerges into a more proactive role, the intranet offers a highly effective way of pushing information at the user.

An intranet is best described as our own internal version of the internet, using the same protocols and browsers as used by the internet. Intranets are going to become increasingly popular in the future as organisations realize how effective they can be.

Most libraries today probably have some form of computer network. An intranet can be viewed as simply a logical extension of the trend in libraries towards greater connectivity. Part of its charm is that it relies mainly on existing technology and infrastructure. For a library or organisation that already has internet access, an intranet is a clever application that more fully exploits features of the internet. With its relatively low cost and ease of set up, the many advantages of an intranet to a library makes it difficult for it to be ignored.

\section{REFERENCES}

1. Bernard, Ryan. Corporate intranet: Create and manage an internal web for your organisation. Wiley, New York, 1996. $p 4$.

2. Ellsworth, J. H. \& Ellsworth, M. V. Internet business book. IASLIC Bulletin, 1998, 43(4),157-62.

3. Griffiths, Peter. Managing your internet and intranet services: The information and library professionals' guide to strategy. Library Association Publishing, London, 2000. pp 161-74.

4. Guha, Suparna \& Adhikari, Abha. New information toolkit for corporate sector. Annals of Library Science and Documentation, 1991, 38(1), 1-7.

5. Hinriches, Randy J. Intranets: What's the bottom line. Sun Microsystems Inc., California, 1997. pp 11-12, 37-38.

6. http://jimmy.qmuc.ac.uk/usr/im94jone/abs tract.htm

7. http://www.ala.org/work/international/zah er.html

8. http://www.ariadnc.ac.uk/issue6/intrnets/

9. http://www.infotoday.com/online/OL1999/ net1.html

10. http://www.slis.ualberta.ca/598/clement/in tro.htm

11. http://www.storm.com/pubwork/intranetp. $\mathrm{html}$

12. Leon, Alexis \& Leon, Mathews. Fundamentals of information technology. Leon Techworld, Chennai, 1999. pp 23.123.7. 
13. Scammell, Alison (Ed.). Handbook of special librarianship and information work. Library Association Publishing, London, 2000. pp 161- 174.

14. Shanmugam, A \& Rajeev S. Computer communication networks. ISTE, New Delhi, 2001. pp 4.72, 9.42.
15. Vishwanath, T. Application of information technology in libraries. Ed. 7. Aslib, London, 1997. pp 6-7, 412, 218, 298.

Contributor: Sh. Bhojaraju G., Library-cum-Information Officer, Indian Rubber Manufacturers Research Association, Thane - 400604.

e-mail: bhojarajug@yahoo.com; http://www.bhojarajug.cjb.net

\section{Readers' Forum}

\section{DearReaders}

We welcome the readers of DESIDOC Bulletin of Information Technology (DBIT). We intend to start a column entitled "Readers' Forum" in which the comments and suggestions of the readers regarding the coverage, quality of papers and other aspects of DBIT will be published.

This will help in further improving the bulletin and making it more educative and useful.

Readers of DBIT are invited to write to the Editor, DBIT on aspects mentioned above. They can send their comments by e-mail also at dirdesidoc@vsnl.net 